\title{
MULTI-AGENT SIMULATION OF HUMAN ACTIVITY FOR RESIDENTIAL ELECTRICAL LOAD AND DEMAND FORECASTING AND ITS APPLICATION TO ELECTRIC MOBILITY
}

\author{
Quentin Reynaud ${ }^{1}$, Mathieu Schumann ${ }^{2 *}$, François Sempé $e^{3}$, Yvon Haradji ${ }^{2}$, Nicolas \\ Sabouret ${ }^{4}$ \\ ${ }^{1} Q R C I$, Clermont-Ferrand, France \\ ${ }^{2} E D F R \& D$, Boulevard Gaspard Monge, Palaiseau, France \\ ${ }^{3}$ FSCI, Paris, France \\ ${ }^{4}$ LISN, Univ. Paris-Sud, Univ. Paris-Saclay, Orsay, France \\ *mathieu.schumann@edf.fr
}

Keywords: Multi-Agent simulation, human activity, residential load and demand forecasting, electric mobility

\begin{abstract}
We present our agent-based architecture focusing on occupant behavior for energy consumption simulations in the residential sector. Based on cognitive ergonomics work, it is aiming at a holistic modelling of human activity, considered as the driver of energy consumption. In our simulations, each household's occupant decides its own actions thanks to a decisional process that puts multiple aspects of human activity in balance and is focused on the interactions between individuals and their material and social environment. This decisional process and this interaction with the environment are guided by the individual's knowledge, preferences and goals, giving the occupants the ability to react and adapt themselves to changes such as electricity price, new appliances or special events. To illustrate the aggregative ambition of the architecture, we present how mobility and actions related to the charging of electric vehicles (EV) were naturally added to the architecture. This new model, centered on both EV use and charging behaviors, aims at assessing the impact of EV on the residential consumption and the electricity distribution network. Finally, the validation processes of our architecture as well as the preliminary validation of the EV model are presented.
\end{abstract}

\section{Introduction}

Societal, political and individual choices related to energy are evolving worldwide towards the reduction of carbon emissions. A visible consequence of this change is the increasing interest in individual electric vehicles (EV) and its impact on local, city or country-scale energy consumption. It is also largely acknowledged that in the residential sector, occupant behavior (OB) has a major influence on energy consumption and has to be included in residential load forecasting studies [1]. Given the notable impact of the acquisition of an EV on a household's load curve, as well as the challenges of a massive development of EV fleets for the grid, a residential energy consumption model can no longer undertake prospective studies without taking EV use into account, both at a local and aggregated scale. The modelling of EV, combined with a residential energy simulation, allows to assess if EV charging loads will generate congestion at bottlenecks in the distribution network, and to evaluate the effectiveness of demand side management or demand response in shaping EV charging load profiles.
Nevertheless, to achieve these goals, the occupant behavior models (OBM) should exhibit some crucial features. In the next section, we will study the main scientific issues identified in both $\mathrm{OB}$ and EV fields. We will then present our holistic approach to OBM and residential energy consumption assessment, and how it is extended to electric mobility. In the same time, we will discuss how this approach brings answers to the identified limits of previous models. In section 3 , we will give a complete overview of our model's general architecture and its application to the Metropolitan French residential sector. Before concluding, section 4 will detail the several validation approaches that were carried out.

\section{State of the art}

\subsection{Occupant Behavior Models}

The OBM field has recently become a research subject receiving close review and intense research effort [2], [3]. The published review papers specify various issues that still need to be tackled. Particularly, [1] call for an OBM that incorporates social, and psychological science to 
reveal the intrinsic causes of OB. Similarly, [4] advocate for OBM that integrate domain experts and modeling methods from building science, social science and psychology to simulate the root cause of occupant behavior on energy consumption. Despite their thorough OBM review, [5] still find a lack in the literature for OBM able to explain and predict occupants' behavior and their interactions with their environment based on multiple aspects of human activity (e.g. conjointly considering activities at home, travels, comfort and thermostat adjustment, appliance use, etc.). Similarly, according to [6], most approaches usually treat each aspect of behavior separately, and individuals of a same household are usually considered independent of each other. We believe that such hypotheses limit the realism and variability of OBM and limits its capacity to react and adapt. To tackle these issues, agent-based models have been identified as good candidates, based on their capacity to integrate knowledge from social and psychological sciences, and to simulate variable, reactive and adaptive behaviors [6].

\subsection{Electric Vehicles models}

Electric vehicle are a relatively new in the mobility landscape, but there are already a variety of approaches in the electric vehicles literature. "Vehicule Ownership and Annual Mileage Models" (VOAMM) are specifically designed to generate EV penetration scenarios, analyze the relations between households' feature and EV presence, as well as between households' features and annual mileage. However, they are not suited for the study of charging patterns in time nor the impact of charging demand peaks on power grids [7].

Within "daily pattern models", activity-based approaches seem to be the more promising ones thanks to their flexibility and their high variability [7]. They consider consistent daily activity-travel schedules to model EV daily use and charging patterns. They are divided in 2 categories: Direct Use of Observed Activity-Travel Schedule (DUOATS) and Activity-Based Models (ABM). In DUOATS, use patterns of cars are used to simulate EV use patterns. Their major limit is their inherent rigidity because of the use of fixed EV travel schedules and predetermined charging behavior scenarios.

[7] advocate for ABM as the most attractive methods because they capture inter-dependence between activity and mobility patterns. They can also model the response to new charging electricity tariffs, and their bottom up structure allows more flexibility and patterns variability than the other approaches. Yet, ABM still have limits to overcome [7]: charging behaviors are rarely explicitly modelled, but usually fixed by scenario. The most common scenario being "uncontrolled charging": the charging operation starts as soon as vehicles reach locations with charging opportunities and is carried out until the vehicle is fully charged or leaves. In other scenarios such as the "delayed or night charging" scenarios, the charging start in the evening, to ensure minimized electricity costs. This use of fixed charging behavior scenarios (mainly due to the lack of available data about real charging behaviors) instead of explicit models is a strong limitation, because it implies predetermined outcomes of demand management policies and prevents the modelling of adaptive charging behaviors.

\subsection{Our proposal to overcome these limits}

Our goal is to develop a single model able to overcome the limits stated in 2.1 and 2.2. In our opinion, the best option is to develop an $\mathrm{ABM}$, based on cognitive ergonomics work focused on human activity, and calibrated with real data. We defend a holistic vision of human activity (i.e. actions and interactions performed by people over time), where all actions are dependent from each other, each of them depicting a part of the global human activity. A single decisional process dynamically attributes weights to every possible action to put them in balance. This decision process has an aggregative goal, since it aims at combining as many different aspects of human activity as possible. This makes it possible to add new actions or models to this architecture to describe new aspects of human activity: for instance electric mobility and charging behaviors. Furthermore, since individual preferences, local environment, tariff policies and non-price-related incentives are considered in the decisional process, it allows the EV charging behaviors to emerge and adapt from each particular situation.

\section{The SMACH model}

SMACH (Simulation of Activity and energy Consumption in Households) is an AI-based multi-agent simulation platform of human activity, applied to the assessment of residential electrical consumption. SMACH considers human activity as the determining factor of energy consumption [8].

\subsection{Population Generation}

First, we use a classical population synthesis methodology [11] to generate households that are statistically representative of the targeted population's characteristics. These include: family structure; age, gender, socioprofessional category, professional situation of individuals; type, surface and thermal performance of buildings; type and energy of heating and domestic hot water; electricity tariff; geography (location, type of urban area, etc.) and weather data. Appliances and their technical characteristics are distributed according to the available data. Currently, the data used is taken from the French national institute of statistics and economic studies (INSEE), as well as specific field surveys.

\subsection{Agent Model}

Each person of the households is modeled as an intelligent agent with goals (its "provisional activity schedule" as described below), knowledge (e.g. the other agents' activities, the price of energy and the state of his local 
environment) and preferences (e.g. comfort temperatures based on quantitative surveys, energy policy oriented towards economy or comfort based on qualitative surveys). For each simulated individual, we use the data from the latest French TUS produced in 2009-2010 by the INSEE to generate a weekly "provisional activity schedule". It is a list of activities to perform, with an estimated duration and a preferential period of the day for carrying them out.

Then, during the simulation, each agent has an individual decision module that will lead to the action that is actually performed. Agents decide themselves the action to perform by pondering his goals, knowledge and preferences, with a 1-minute time step (please refer to [9], [10] for a complete presentation of the agent model and its decision module). Our agents are thereby able to modify their actions, for instance according to the actions of others (e.g. "My regular schedule is to take my shower first and then to have lunch, but the shower is currently occupied so I take my breakfast first"), or according to other factors such as the price of energy or the weather conditions.

\subsection{Expanding the range of simulated activities: the SMACH mobility model}

With this agent model as a basis, we expanded the architecture to include individual mobility and EV charging. In accordance with [12] demonstrating that an EV model built with help of household travel surveys (HTS) can accurately reproduce EVs' driving behaviors, we choose the last available French HTS (2010 "National Transport and Travel Survey") to calibrate our EV model. Additional French data focused on EV ownership, type of driving and recharging behaviors were also used [13]. This second set of data allows us to fulfill the first step of the model's expansion to integrate EV, namely adding EV ownership parameters in the population generation process. These details concern: which individual possesses an EV (and its brand and model), its type of driving (standard or eco driving), the type of home charging station (none, $2,3.2,3.7$, or $7.4 \mathrm{~kW}$ ), and the presence of a public charging station usable near home. In the EV attribution process, the following individual characteristics are taken into account: age and gender, type of household and size, type of housing, type of urban area.

We then created a specific mobility model associated with SMACH's activity-centered approach. While TUS data describe activities (duration, location, etc.), HTS data describe car-traveled distances to go from a location to another. Therefore, we coupled these two logics with help of a common typology of locations. In the current version of our model, we consider five locations (home, work, shopping, leisure and "other") and locate each TUS-related activity in one of them (i.e. all the shopping related activities take place in the "shopping" location, etc.).

Then, we use the HTS data to compute, for each "HTS individual" (a real respondent in the HTS database), the car distance matrix of these five locations, i.e. the average distance travelled by car to reach all these locations from any other (the averaging allowing to consider all travels, even the ones that do not use the car). For each simulated individual driving an $\mathrm{EV}$, we assign the car distance matrix from a real "HTS individual" sharing the same individual characteristics as our "TUS individual", and thereby determine the distance travelled by car each time the individual changes location by car.

Each EV is modelled as an agent with the following features: brand and model, maximum and current battery capacity, current location, and energy consumption per km in urban and rural areas $(\mathrm{kWh} / \mathrm{km})$. These consumptions per $\mathrm{km}$ are constant and computed beforehand using an EV consumption model taking their weight, frontal area, drag coefficient, efficiencies of the electronics, motor and transmission components, brake regeneration, WLTC driving cycle, and type of driving into account. The type of WLTC used depends on the household's geographical location (see 3.1).

Our EV model also integrate an explicit charging behavior model. Figure 1 from [13], gives an example of charging options and statistics in four locations, considering some household features such as the type of housing and the type of urban area.

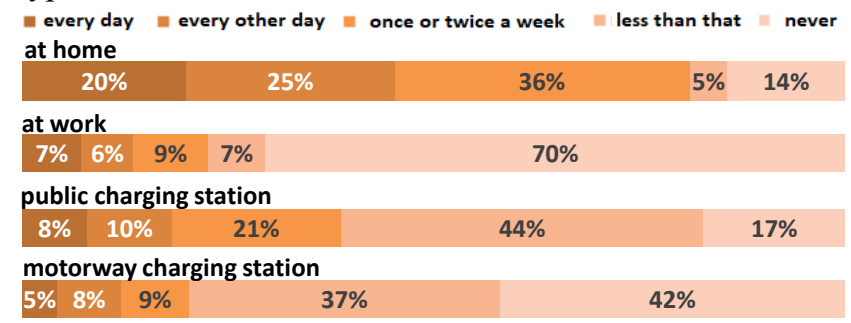

Figure 1: Example of charging behaviors distribution

From this data, we implemented a type of charging behavior for each location, related to the current $\mathrm{EV}$ charging trends. However, the charging patterns are not fixed, since the charging possibilities depend on the vehicle location, which is decided by the human decisional process (not related with HTS data, but with TUS data). In addition, the current battery capacity affects the people's charging decisions, and the electricity tariffs can also have a direct impact, as the home charging stations are often linked to off-peak hours or a dedicated tariff. Charging times cannot exceed the time that the vehicle spends in a given location. Charging power of public charging stations was inferred from GIREVE database (www.gireve.com). To sum up, our EV model is composed of a "VOAMMlike" model used to determine EV ownership details, an Agent-Based Model for EV-use based on Household Travel Surveys data (HTS), and an explicit charging model that combine HTS, TUS and field study data. These three models benefit from the emerging and adaptive capacities of the architecture. Indeed, EV related actions are placed at the same level as every other aspect of human activity, and the simulated individuals adapt themselves to changes in the environment (tariff, weather, specific events, etc.), thanks to their individual decisional process. 


\subsection{Application to the assessment of residential load curves and energy use}

We consider energy consumption as a consequence of human activity. Thus, SMACH associates human actions and decisions with four categories of appliances: controlled by a setpoint or a tariff (including heating and domestic hot water), programmed (e.g. a dishwasher programmed to start at a given time), linked to an activity (e.g. a TV for the activity watching TV), and constant use (e.g. an internet router). The newly introduced EV charging model can fall into the first 3 categories, depending on the charging behavior of the user and the charging control devices they possess. It is also possible to model interaction between energy uses, for instance if a household prefers to give precedence to producing enough hot water over charging their EV.

Each appliance has a specific active and reactive power demand model, which can be a constant power over time, the result of a dynamic thermal model (e.g. for the domestic hot water), or a curve based on laboratory measurements (e.g. for washing machine cycles). Heating and cooling needs are computed by a previously-validated dynamic building energy model, built from the BuildsysPro Modelica library, and co-simulated with SMACH using the FMI interoperability standard [14].

SMACH continuously meters the use and power load of each appliance to calculate the load curve of the household. For large scale assessments, a target population is generated by $\mathrm{SMACH}$ (see 3.1), and the global residential load curve is obtained by simulating each individual household and aggregating the individual results.

\section{Discussion on validations}

To take the specificity of a human-centered agent model into account, we devised and performed a dedicated validation process at both household and aggregated level, considering both human activity and energy consumption.

\subsection{Validation at the individual household scale}

We validated the simulated activity with the implementation of a specific cognitive ergonomics approach known as 'participatory simulation' [15], carried out with 10 families participating in the "OpCo" demonstrator in Brittany, France. A dedicated HumanComputer Interface was developed to allow real people to manipulate SMACH and judge themselves the realism of their own household simulation. The results proved our model to be able to faithfully reproduce human activity at an individual level, with its complexity and its own internal logic (daily life, interactions, cooperation) [16].

The SMACH results were also compared to the metered load curves of the OpCo households over 8 to 15 weeks at a $30 \mathrm{~min}$ time step. The simulated load curves showed to be within a satisfying confidence interval compared to the OpCo curves, with mean errors on energy consumptions ranging between $2 \%$ and $14 \%$ (Figure 2).

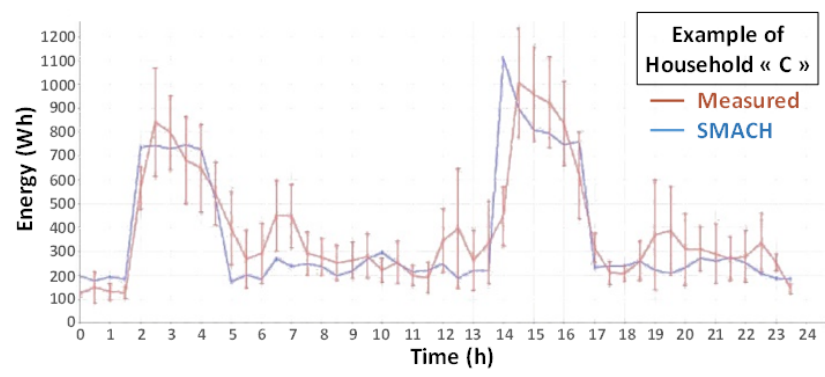

Figure 2: Confidence interval comparison for Tuesdays

\subsection{Validation at the aggregated scale}

We verified that our simulations reproduced relevant indicators at the aggregated level when compared to the INSEE data, including, for each action, average activities' durations, number of repetitions, preferred realization periods, which were adequately close to the statistical data. To ensure that the SMACH results agreed on the main characteristics of residential consumption, we generated a population of 1,000 households representative of Metropolitan France and compared the aggregated load curve with the annual and weekly profiles used for the assessment of injection and withdrawal flows on the public distribution network (DSO open data). This data was used to validate the dynamics and weekly values of the simulated load curves (Figure 3 and Figure 4), and helped identify the need to better take the dynamics of heating systems such as heat pumps into account.

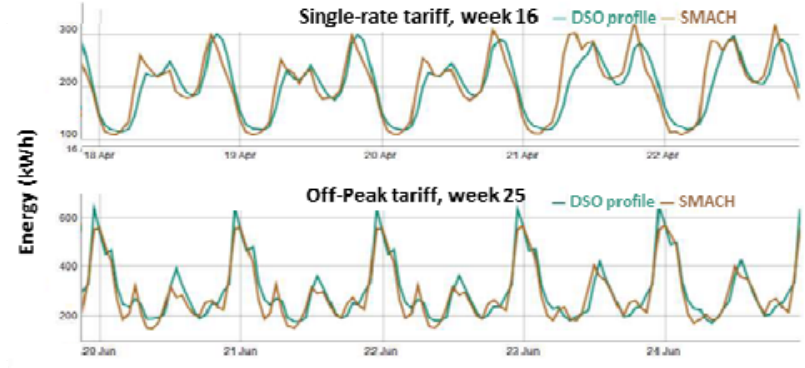

Figure 3: Load curve for Single-Rate and Off-Peak tariffs



Figure 4: Comparison of weekly energy consumptions for one year under the "peak/off-peak tariff" for mean meteorological data (left) and the cold year 2012 (right)

\subsection{EV model preliminary validation}

As a first validation step, we generated a virtual population composed of 10000 household, and verified that the EV ownership details were similar than those in data set (e.g. on average $1 \mathrm{EV}$ for 330 households). Then, we simulated 
this population during one year and compared the EV-use with HTS data. We obtained an average $12700 \mathrm{~km}$ against the $12900 \mathrm{~km}$ in the data. Figure 5 details the average distance traveled during weekend in HTS data compared to simulations. The low representativeness of high mileage in our simulations comes from the current absence of "long trips" in the model, which are currently being included.

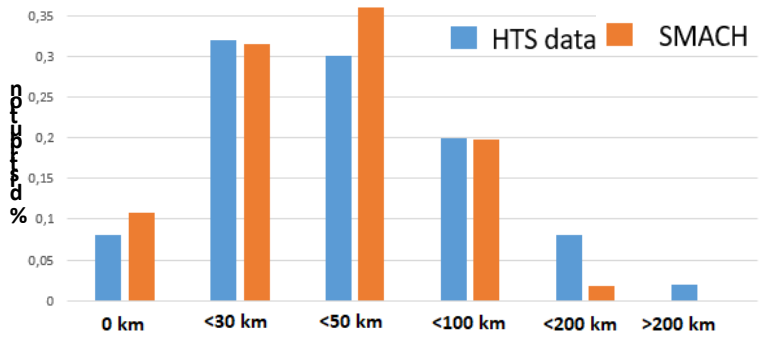

Figure 5: Distribution of EV trips during weekends

Currently, the validation at the individual level were only performed through expert verifications, to ensure that the simulated EV-use is consistent with individual HTS data.

\section{Conclusion and perspectives}

We presented a generic agent architecture, aiming at a holistic modelling of human activity, based on cognitive ergonomics work. This architecture was applied to residential energy consumption simulations, allowing the generation of coherent load curves at both individual and aggregated levels. This model was extended to simulate EV use and charging profiles for each EV present in a given population. This EV model still lacks validation since large and good quality data sets are difficult to obtain, but we already verified its ability to reproduce aggregated data from an emergent bottom-up perspective.

Our agent architecture has been in constant enhancement for more than 10 years, and the newly added EV module has many areas of improvements. The most critical being certainly the collective organization inside a household around the EV use. Indeed, in the current version, each EV is driven only by one human, which oversimplifies the way people organize their trips and decide on EV charging.

Applications to grid issues encompass prospective impact studies of EV market penetration on peak loads at a global and local level, as well as studies on interactions between grid constraints and residential consumption from a V2G perspective. For end consumers, this model also supports the development and sizing of smart charging solutions, or the shaping of new customized electricity tariffs.

\section{References}

[1] J. Li, Z. J. Yu, F. Haghighat, and G. Zhang, "Development and improvement of occupant behavior models towards realistic building performance simulation: A review," Sustain. Cities Soc., vol. 50, p. 101685, 2019.

[2] W. O'Brien et al., "An international review of occupant-related aspects of building energy codes and standards," Build. Environ., p. 106906, 2020.

[3] W. O'Brien et al., "Introducing IEA EBC Annex 79: Key challenges and opportunities in the field of occupant-centric building design and operation," Build. Environ., p. 106738, 2020.

[4] F. D. Salim et al., "Modelling urban-scale occupant behaviour, mobility, and energy in buildings: A survey," Build. Environ., vol. 183, p. 106964, 2020.

[5] S. Carlucci et al., "Modeling occupant behavior in buildings," Build. Environ., p. 106768, 2020.

[6] G. Happle, J. A. Fonseca, and A. Schlueter, "A review on occupant behavior in urban building energy models," in Energy and Buildings, vol. 174, Elsevier Ltd, 2018, pp. 276-292.

[7] N. Daina, A. Sivakumar, and J. W. Polak, "Modelling electric vehicles use: a survey on the methods," Renew. Sustain. Energy Rev., vol. 68, pp. 447-460, 2017.

[8] E. Amouroux, T. Huraux, F. Sempé, N. Sabouret, and Y. Haradji, "Simulating human activities to investigate household energy consumption," in Proceedings of the 5th International Conference on Agents and Artificial Intelligence, 2013, vol. 2, pp. 71-80, Accessed: Sep. 21, 2016. [Online]. Available: http://perso.limsi.fr/sabouret/ps/icaart2013.pdf.

[9] Q. Reynaud, Y. Haradji, F. Sempé, and N. Sabouret, "Using Time Use Surveys in Multi Agent based Simulations of Human Activity.," in ICAART (1), 2017, pp. 67-77.

[10] Q. Reynaud, N. Sabouret, Y. Haradji, and F. Sempé, "Simulation de l'activité humaine. Une étude sur le réalisme multi-niveau," Rev. d'Intelligence Artif., vol. 32, pp. 197-221, 2018.

[11] K. Müller and K. W. Axhausen, "Population synthesis for microsimulation: State of the art," 2011.

[12] G. Pareschi, L. Küng, G. Georges, and K. Boulouchos, "Are travel surveys a good basis for EV models? Validation of simulated charging profiles against empirical data," Appl. Energy, vol. 275, p. $115318,2020$.

[13] J. Cubillé, "Principaux résultats de la première vague du Baromètre des Acquéreurs de VE et de VHR (BdAVE) : France et Italie,” 2019.

[14] G. Plessis, E. Amouroux, and Y. Haradji, "Coupling occupant behaviour with a building energy model - A FMI application," in 10th International Modelica Conference, Mar. 2014, vol. 96, pp. 321-326, doi: 10.3384/ecp14096321.

[15] Y. Haradji, G. Poizat, and F. Sempé, Human activity and social simulation. Boca Raton, FL : CRC Press, 2012.

[16] Y. Haradji et al., "From modeling human activity to modeling for social simulation: between realism and technological innovation," Act. Electron., vol. 15 , no. $1,2018$. 